\title{
Self-similar focusing with generalized devil's lenses
}

\author{
Cristina Casanova, ${ }^{1}$ Walter D. Furlan, ${ }^{1}$ Laura Remón, ${ }^{2}$ Arnau Calatayud, ${ }^{2}$ \\ Juan A. Monsoriu, ${ }^{2, *}$ and Omel Mendoza-Yero ${ }^{3}$ \\ ${ }^{1}$ Departamento de Óptica, Universidad de Valencia, E-4610o Burjasstot (Valencia), Spain \\ ${ }^{2}$ Centro de Tecnologías Físicas, Universidad Politécnica de Valencia, E-46022 Valencia, Spain \\ ${ }^{3}$ GROC UJI, Departament de Física, Universitat Jaume I, E12080 Castelló, Spain \\ ${ }^{*}$ Corresponding author: jmonsori@fis.upv.es
}

Received September 28, 2010; accepted November 21, 2010;

posted November 30, 2010 (Doc. ID 135832); published January 26, 2011

\begin{abstract}
We introduce the generalized devil's lenses (GDLs) as a new family of diffractive kinoform lenses whose structure is based on the generalized Cantor set. The focusing properties of different members of this family are analyzed. It is shown that under plane wave illumination the GDLs give a single main focus surrounded by many subsidiary foci. It is shown that the total number of subsidiary foci is higher than the number of foci corresponding to conventional devil's lenses; however, the self-similar behavior of the axial irradiance is preserved to some extent. @ 2011 Optical Society of America

OCIS codes: $\quad 050.1940,050.1965$.
\end{abstract}

\section{INTRODUCTION}

Diffractive focusing optical elements are most beneficial in many applications where they can perform tasks that are difficult, or even impossible, with conventional refractive optics [1]. In particular, nowadays diffractive lenses are key elements in optical systems used in several scientific and technological areas such as THz tomography, astronomy, and soft x-ray microscopy [2-5]. Aside from conventional Fresnel zone plates, other geometries of photonic-image-forming structures have been proposed. One of them is known as a fractal zone plate (FZP) [6]. A FZP is characterized by its fractal profile along the square of the radial coordinate. The main feature of a FZP arises from its ability to produce multiple foci. The main lobe of these foci coincide with that of the associated conventional zone plate, but the internal structure of each focus exhibits a characteristic fractal structure, reproducing the self-similarity of the originating FZP. Note that, under certain circumstances, an FZP can be thought of as a conventional zone plate with certain missing zones [6].

Since their introduction in 2003, FZPs have deserved the attention of several research groups working on diffractive optics [ㄱ,,$\underline{8}$. This is mainly because in certain applications they can improve the performance of classical Fresnel zone plates, especially where multiple foci are useful. For example, we can profit= from FZPs by using them in image forming systems working under polychromatic illumination [9], using them as versatile optical tweezers [10], for designing novel optical filters with fractal transmission spectra [11], and as encrypting masks in optical cryptography [리].

In order to improve the diffraction efficiency of FZPs for certain applications, we have recently proposed pure phase FZPs known as devil's lenses (DLs) [13]. In particular, the surface relief of a DL is designed using the devil's staircase function [14]. Contrary to FZP a DL presents a single principal focus surrounded by subsidiary secondary foci, following a self-similar distribution. An experimental implementation of a DL having multilevel phases has been recently reported [15].
In this work, we analyze the focusing properties of the novel optical devices called generalized devil's lenses (GDLs), which are constructed from the "generalized" Cantor set. The blazed profile of a GDL improves the diffraction efficiency of a DL with respect to that of the generalized FZP [16]. The focusing properties of different GDLs are studied by computing their irradiance distribution along the optical axis. In addition, with the introduction of a generalization parameter, the number of subsidiary foci is increased in comparison with triadic DLs. Its effect on the self-similar focusing properties is investigated.

\section{GENERALIZED DEVIL'S LENSES DESIGN}

The original proposal for a DL [13] was based on the triadic Cantor set shown in the upper part of Fig. 1 . The first step in the construction procedure consists of defining a straight-line segment of unit length called initiator (stage $S=0$ ). Next, at the stage $S=1$, the generator is constructed by dividing the segment in three equal parts of length $1 / 3$ and removing the central one. Following this procedure in subsequent stages $S=2,3, \ldots$, it is easy to see that, in general, at the stage $S$ there are $2^{S}$ segments of length $d=3^{-S}$ with $2^{S-1}$ disjoint gaps. In Fig. 1, only the three first stages are shown for clarity. It is instructive to note that the Cantor set at a given stage $S$ is interpreted as a quasi-periodic distribution of segments, which can be obtained by removing some segments in a finite periodic distribution. The equivalent periodic distribution at the stage $S$ has $(3 S-1) / 2+1$ segments of length $3^{-S}$, separated by gaps of the same length, so the period of this finite structure would be $\Lambda=2 \times 3^{-S}$.

Following the above iterative process we can define a more general triadic fractal Cantor set in which the ratio between lengths of the equivalent period $(\Lambda)$ and of the segment $(d)$ is a positive integer number $\tau=\Lambda / d$. We call this the generalization parameter of the Cantor set. According to the above definition, a conventional (triadic) Cantor set is a particular case with $\tau=2$. Thus the generalized Cantor set can be obtained by 


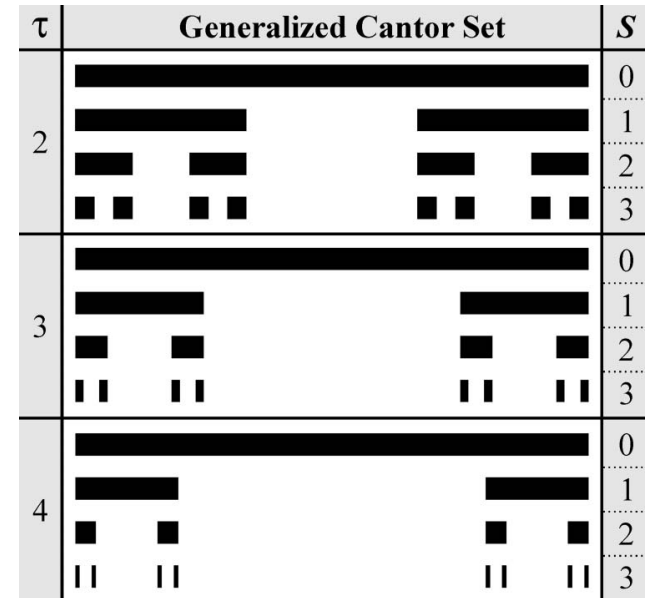

Fig. 1. Generalized Cantor set for $\tau=2,3$, and 4 and growth levels $S=0,1,2$, and 3 .

dividing the initiator into $(\tau+1)$ parts and removing $(\tau-1)$ central segments. This operation yields a family of generalized Cantor sets with equivalent period $\Lambda=\tau(\tau+1)^{-S}$. Therefore, generalized Cantor fractals are characterized by the generalization parameter $\tau$ and the growth level $S$. In general, at the stage $S$ there are $2^{S}$ segments of length $(\tau+1)^{-S}$ with $2^{S}-1$ disjoint gap intervals $\left[p_{\tau, S, l}, q_{\tau, S, l}\right]$, with $l=1, \ldots, 2^{S}-1$. For example, for $\tau=3$ and $S=3$ (see Fig. 1), the generalized Cantor set presents seven gaps limited by the following positions inside the unit length: $[1 / 64,3 / 64],[4 / 64,12 / 64],[13 / 64,15 / 64]$, $[16 / 64,48 / 64],[49 / 64,51 / 64],[52 / 64,60 / 64]$, and $[61 / 64$, $63 / 64]$. Based on the generalized Cantor set, we can define now the generalized Cantor function or generalized devil's staircase, $F_{\tau, S}(x)$, in the interval $[0,1]$ as

$$
F_{\tau, S}(x)=\left\{\begin{array}{cc}
\frac{l}{2^{S}} & \text { if } p_{\tau, S, l} \leq x \leq q_{\tau, S, l} \\
\frac{1}{2^{S}} \frac{x-q_{\tau, S, l}}{p_{\tau, S, l+1-q_{\tau, S, l}}}+\frac{l}{2^{S}} & \text { if } q_{\tau, S, l} \leq x \leq p_{\tau, S, l+1}
\end{array},\right.
$$

being $F_{\tau, S}(0)=0$ and $F_{\tau, S}(1)=1$. Figure 2 shows the generalized devil's staircase for $\tau=3$ and growth levels $S=0,1,2$, and 3 . Note that within the gap intervals $\left[p_{\tau, S, l}, q_{\tau, S, l}\right]$, the generalized devil's staircase, $F_{\tau, S}(x)$, takes the constant values $l / 2^{S}$ with $l=1, \ldots, 2^{S}-1$, whereas in between these intervals it increases linearly.

From the generating function $F_{\tau, S}(x)$ we define the corresponding GDL as a circularly symmetric diffractive optical element with a phase profile that follows the generalized Cantor function. At the gap regions defined by the generalized Cantor set, the phase shift is $-l / 2 \pi$, with $l=1, \ldots, 2^{S}-1$. Thus, the phase transmittance of a GDL is defined by

$$
\begin{aligned}
q(\varsigma) & =q_{\mathrm{GDL}}(\varsigma, \tau, S)=\exp \left[\mathrm{i} \phi_{\tau, S}(\varsigma)\right], \\
\text { with } \phi_{\tau, S}(\varsigma) & =-2^{s+1} \pi F_{\tau, S}(\varsigma),
\end{aligned}
$$

where $\varsigma=(r / a)^{2}$ is the normalized quadratic radial variable and $a$ is the lens radius. Figure 3(a) shows the phase transmittance $\phi_{\tau, S}$ for $\tau=3$ and $S=2$ versus the quadratic radial variable $\varsigma$. After taking $\bmod _{2 \pi}\left[\phi_{\tau, S}\right]$, the equivalent phase profile is shown in Figs. 3(b) and 3(c) versus the quadratic radial variable $\varsigma$ and versus the radial coordinate $r$, respectively. Thus, at each zone of the lens the phase variation is quadratic. The profile of the surface relief, $h_{\mathrm{GDL}}(r)$, corresponding to the above phase function can be obtained from the relation [17]
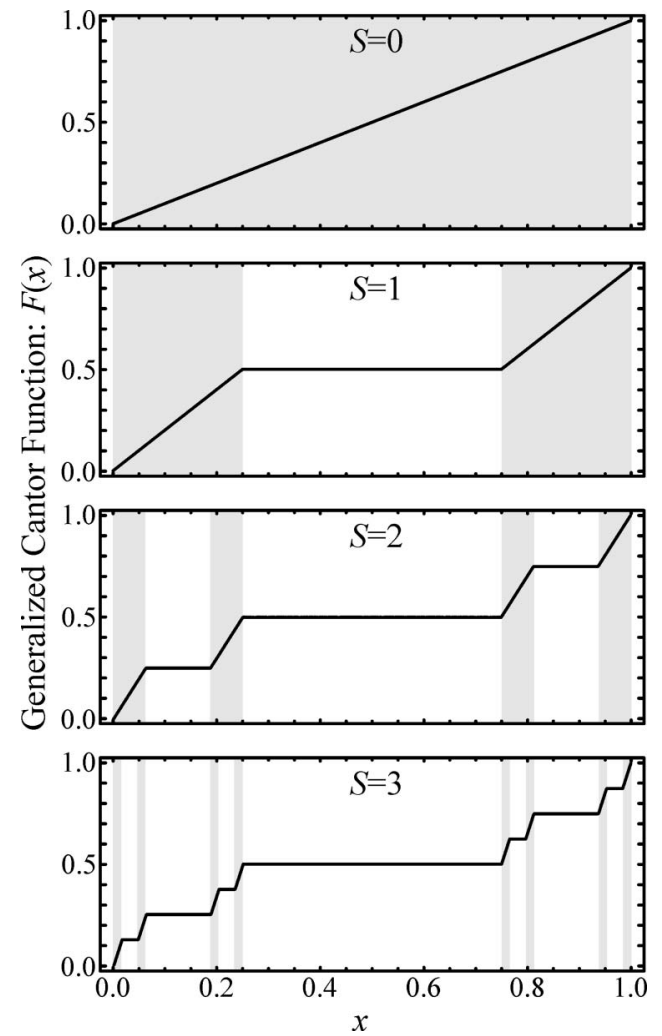

Fig. 2. Generalized devil's staircase function for $\tau=3$ and growth levels $S=0,1,2$, and 3 . The gray bars represent the corresponding generalized Cantor set.

$$
h_{\mathrm{GDL}}(r)=\bmod _{2 \pi}\left[\phi_{\tau, S}\left(\frac{r^{2}}{a^{2}}\right)\right] \frac{\lambda}{2 \pi(n-1)},
$$

where $n$ is the refractive index of the optical material used for constructing the GDL, and $\lambda$ is the wavelength of the light.

Note that the GDL can be understood as a conventional kinoform lens but with some missing phase zones (see lower part of Fig. 3). In fact, for a given generalization parameter

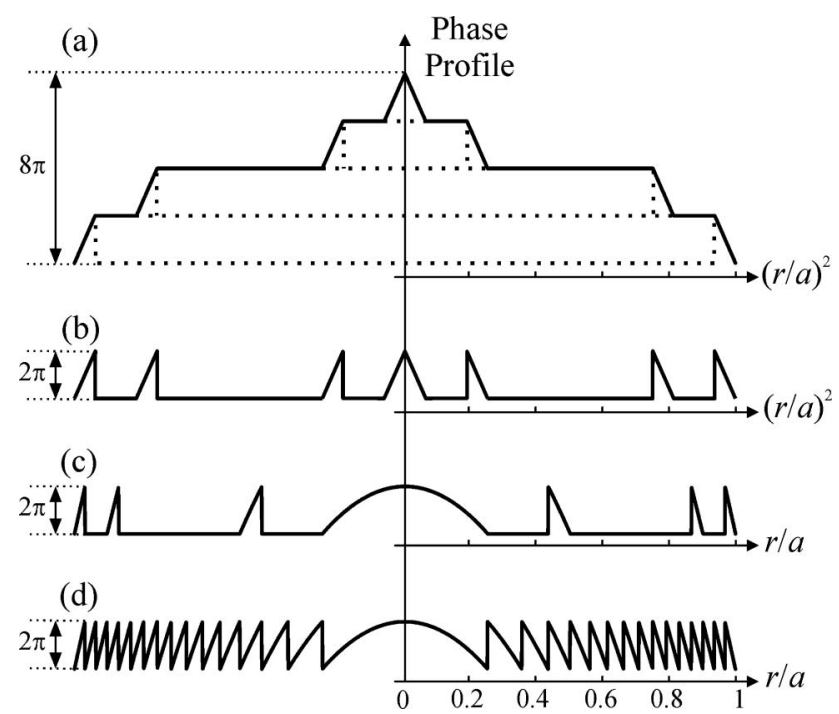

Fig. 3. (a) Phase transmittance of a GDL for $\tau=3$ and $S=2$ versus the quadratic radial variable. (b) Equivalent phase transmittance after taking modulus $2 \pi$. (c) Phase transmittance versus the radial variable. (d) Phase transmittance of a kinoform lens of the same focal length. 
$\tau$ and growth level $S$, we can perform a GDL from a conventional kinoform lens with $(\tau+1)^{S}$ blazed phase zones by removing the $(\tau+1)^{S}-2^{S}$ zones located at the gaps of the generalized Cantor set. Note that the phase transmittance of a DL is obtained when $\tau=2$.

\section{FOCUSING PROPERTIES OF GENERALIZED DEVIL'S LENSES}

Let us consider the irradiance provided by a rotationally invariant pupil of radius $a$, and amplitude transmittance $p(r)$, at a given point on the optical axis. This pupil is illuminated by a monochromatic plane wave of wavelength $\lambda$. Within the Fresnel approximation, the axial irradiance can be expressed as a function of the axial distance from the pupil plane $z$, as

$$
I(z)=\left(\frac{2 \pi}{\lambda z}\right)^{2}\left|\int_{0}^{a} p\left(r_{o}\right) \exp \left(-i \frac{\pi}{\lambda z} r_{o}^{2}\right) r_{o} \mathrm{~d} r_{o}\right|^{2}
$$

If the transmittance of the pupil is defined in terms of the normalized variable $\varsigma=(r / a)^{2}$, then the axial irradiance can be rewritten as

$$
I(u)=4 \pi^{2} u^{2}\left|\int_{0}^{1} q(\varsigma) \exp (-i 2 \pi u \varsigma) \mathrm{d} \varsigma\right|^{2},
$$

where $q(\varsigma)=p\left(r_{o}\right)$, and $u=a^{2} / 2 \lambda z$ is the reduced axial coordinate. Thus, the axial irradiance can be determined by the Fourier transform of the mapped pupil function $q(\varsigma)$. From self-similar properties of fractals, if the pupil function $q(\varsigma)$ has a fractal structure, it is direct to conclude that such a system will provide an irradiance along the optical axis also with a self-similar profile [18]. As an example we have computed the axial irradiances produced by GDLs at levels $S=1$ and $S=2$. The result is represented in Fig. 4 for three values of the generalization parameter of the Cantor set: $\tau=2,3$, and 4 . Note that values of the axial coordinate for $S=2$ are scaled by a factor $\gamma=\tau+1$ with respect to $S=1$. The main foci of GDLs are located at the normalized distance $u_{0}=(\tau+1)^{S}$, i.e., the focal length of the GDLs can be expressed as

$$
f_{\tau, S}=\frac{a^{2}}{2 \lambda(\tau+1)^{S}}
$$

Note that the axial positions of the central lobes of the main foci for the GDL coincide with those of the associated kinoform lenses.

From Fig. 4 it is clear that GDL focusing properties are determined by $\tau$, and therefore this parameter imposes the limits of the diffraction efficiency of the lens. It is important to note that as $\tau$ increases, there will also be an increase in the background radiation because of the zeroth diffraction order.

Note that the axial irradiance distribution at the focal region at each stage is a modulated version of that associated with the previous stage. In fact, the patterns in the right part of Fig. 4 are self-similar, i.e., as $S$ becomes larger an increased number of zeros and maxima are encountered. These axial irradiance patterns are scale invariant over dilations of factor $\gamma=\tau+1$. This means that, for a GDL, its axial irradiance reproduces the self-similarity of the pupil.


Normalized axial coordinate: $u$ Normalized axial coordinate: $u$ Fig. 4. Axial irradiances provided by GDL of orders $S=1$ and $S=2$ and generalization parameters $\tau=2,3$, and 4 .

To investigate quantitatively the degree of self-similarity of the axial irradiances provided by a given GDL, we use the correlation coefficient [19] between the axial irradiance and its scaled version with respect to the axial focal point $u_{0}=(\tau+1)^{S}$, i.e.,

$$
C(\gamma)=\frac{\int_{0}^{\infty} I(u) \cdot I\left(u_{0}+\frac{u-u_{0}}{\gamma}\right) \mathrm{d} u}{\sqrt{\int_{0}^{\infty} I^{2}(u) \mathrm{d} u \int_{0}^{\infty} I^{2}\left(u_{0}+\frac{u-u_{0}}{\gamma}\right) \mathrm{d} u}} .
$$

This function was computed for the irradiance of a GDL at the stage $S=2$. When $I(z)$ satisfies the strict axial self-similar

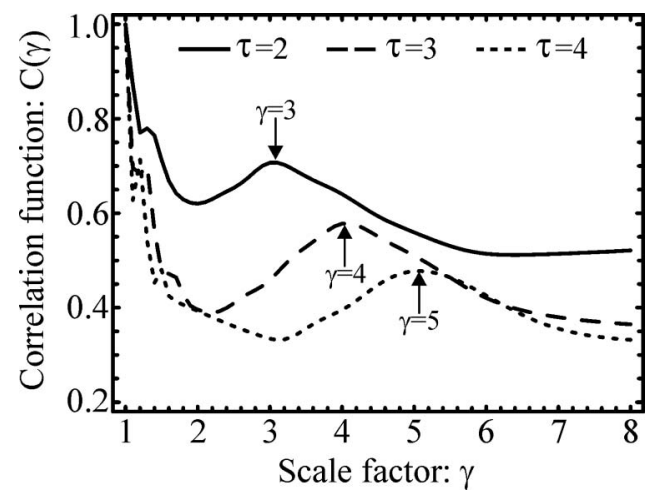

Fig. 5. Self-similarity for the axial irradiance shown in Fig. $\underline{4}$ for $S=2$. 
property, i.e., $I(u)=I\left(u_{0}+\left(u-u_{0}\right) / \gamma\right)$, the correlation coefficient (or simply, the self-similarity) is $C(\gamma)=1$. In the same way, lower degrees of self-similarity correspond to values of $C(\gamma)<1$. In Fig. 5 the correlation function for the GDLs in Fig. 4 , at the stage $S=2$, is represented. Although the correlation coefficient decreases as the generalization parameter $\tau$ increases, we find a local maximum at $\gamma=\tau+1$. This maximum shows the degree of self-similarity of the axial irradiances, which is a consequence of the fractal structure of the GDLs.

\section{CONCLUSIONS}

The generalized devil's lenses designed on the basis of the generalized Cantor function have been introduced. It is shown that a given GDL can be understood as a conventional kinoform lens in which some blaze zones have been eliminated. In addition, the phase transmittance function of a conventional DL is obtained as a particular case of Eq. (2) when $\tau=2$. The GDLs produce a single main focus at which the position of the maximum axial irradiance coincides with that of the corresponding Fresnel kinoform lens. However, in this case the maximum is surrounded by subsidiary foci with self-similar profiles. Along the optical axis, the focal volume reproduces the fractal distribution of the originating GDL. The degree of self-similarity of the axial irradiance provided by these novel lenses was also investigated.

Several potential applications of GDLs are possible, especially in those areas where conventional kinoform lenses have been successfully applied (such as image forming systems or optical trapping). In particular, we suggest that the multifocal structure of a GDL can find application, for instance, for trapping and manipulating particles at different controlled levels by means of a vortex GDL.

\section{ACKNOWLEDGMENTS}

We acknowledge the financial support from the Ministerio de Ciencia e Innovación, Spain, grants DPI2008-02953, TRA20090215, and FIS2010-15746. We also acknowledge the support from Generalitat Valenciana (PROMETEO2009-077 and ACOMP/2010/052), and from Universidad Politécnica de Valencia, Spain (PAID-05-09 and PAID-06-08). L. Remón acknowledges a fellowship of "Fundación Cajamurcia," Spain.

\section{REFERENCES}

1. J. Ojeda-Castañeda and C. Gómez-Reino, eds., Selected Papers on Zone Plates (SPIE Optical Engineering Press, 1996).

2. S. Wang and $\mathrm{X}$. Zhang, "Terahertz tomographic imaging with a Fresnel lens," Opt. Photonics News 13, 59-59 (2002).

3. Y. Wang, W. Yun, and C. Jacobsen, "Achromatic Fresnel optics for wideband extreme-ultraviolet and x-ray imaging," Nature (London) 424, 50-53 (2003).

4. L. Kipp, M. Skibowski, R. L. Johnson, R. Berndt, R. Adelung, S. Harm, and R. Seemann, "Sharper images by focusing soft x-rays with photon sieves," Nature (London) 414, 184-188 (2001)

5. G. Andersen, "Large optical photon sieve," Opt. Lett. 30, 2976-2978 (2005).

6. G. Saavedra, W. D. Furlan, and J. A. Monsoriu, "Fractal zone plates," Opt. Lett. 28, 971-973 (2003).

7. J. A. Davis, L. Ramirez, J. A. Rodrigo Martín-Romo, T. Alieva, and M. L. Calvo, "Focusing properties of fractal zone plates: Experimental implementation with a liquid-crystal display," Opt. Lett. 29, 1321-1323 (2004).

8. H.-T. Dai, X. Wang, and K.-S. Xu, "Focusing properties of fractal zone plates with variable lacunarity: Experimental studies based on liquid crystal on silicon," Chin. Phys. Lett. 22, 2851-2854 (2005).

9. W. D. Furlan and G. Saavedra, and J. A. Monsoriu, "White-light imaging with fractal zone plates," Opt. Lett. 32, 2109-2111 (2007).

10. S. H. Tao, X.-C. Yuan, J. Lin, and R. Burge, "Sequence of focused optical vortices generated by a spiral fractal zone plate," Appl. Phys. Lett. 89, 031105 (2006).

11. O. Mendoza-Yero, G. Mínguez-Vega, M. Fernández-Alonso, J. Lancis, E. Tajahuerce, V. Climent, and J. A. Monsoriu, "Optical filters with fractal transmission spectra based on diffractive optics," Opt. Lett. 34, 560-562 (2009).

12. M. Tebaldi, W. D. Furlan, R. Torroba, and N. Bolognini, "Opticaldata storage-readout technique based on fractal encrypting masks," Opt. Lett. 34, 316-318 (2009).

13. J. A. Monsoriu, W. D. Furlan, G. Saavedra, and F. Giménez, "Devil's lenses," Opt. Express 15, 13858-13864 (2007).

14. D. R. Chalice, "A characterization of the Cantor function," Am Math. Mon. 98, 255-258 (1991)

15. D. Wu, L.-G. Niu, Q.-D. Chen, R. Wan, and H.-B. Sun, "High efficiency multilevel phase-type fractal zone plates," Opt. Lett. 33, 2913-2915 (2008).

16. O. Mendoza-Yero, M. Fernández-Alonso, G. Mínguez-Vega, J. Láncis, V. Climent, and J. A. Monsoriu, "Fractal generalized zone plates," J. Opt. Soc. Am. A 26, 1161-1166 (2009).

17. Y. Han, L. N. Hazra, and C. A. Delisle, "Exact surface-relief profile of a kinoform lens from its phase function," J. Opt. Soc. Am. A 12, 524-529 (1995).

18. C. Allain and M. Cloitre, "Optical diffraction on fractals," Phys. Rev. B 33, 3566-3569 (1986).

19. J. A. Monsoriu, C. J. Zapata-Rodriguez, and W. D. Furlan, "Fractal axicon," Opt. Commun. 263 (1), 1-5 (2006). 